\title{
Group-Based Worry Intervention for Persecutory Delusions: an Initial Feasibility Study
}

\author{
Louise Isham* \\ Department of Psychiatry, University of Oxford, Warneford Hospital, Warneford Lane, Oxford OX3 7JX \\ and Oxleas NHS Foundation Trust, 63 Yeoman House, Penge, London SE20 7TS
}

Heidemarie Grafahrend

Oxleas NHS Foundation Trust, Bridgeways Day Hospital, Turpington Lane, Bromley BR2 8JA

\author{
Alecia Nickless
}

Primary Care Clinical Trials Unit, Nuffield Department of Primary Care Health Sciences, University of Oxford, Radcliffe Observatory Quarter, Woodstock Road, Oxford OX2 6GG

\section{Katherine Pugh}

Sussex Partnership NHS Foundation Trust, Research \& Development Department, Sussex Education Centre, Nevill Avenue, Hove, East Sussex BN3 7 HY

\section{Steve Pleasants}

Oxleas NHS Foundation Trust ResearchNet, 63 Croydon Road, London SE20 7TS

\begin{abstract}
Nicola Smedley
Oxleas NHS Foundation Trust, Bromley Intensive Case Management Psychosis Team East, 1-6 Carlton Parade, Orpington, Kent BR6 OJB

\section{Daniel Freeman}

Department of Psychiatry, Warneford Hospital, University of Oxford, Oxford OX3 7JX
\end{abstract}

* Correspondence to Louise Isham. E-mail: louise.isham @ psych.ox.ac.uk 


\author{
Alison Mulligan \\ Oxleas NHS Foundation Trust, Bromley Intensive Case Management Psychosis Team West, \\ 63 Yeoman House, Penge, London SE20 7TS
}

\begin{abstract}
Background: A one-to-one cognitive behavioural therapy intervention targeting worry significantly reduces both worry and persecutory delusions (Freeman et al., 2015). Aim: To adapt this intervention for group delivery and conduct a feasibility trial within routine clinical practice. Method: Thirteen participants were randomized to a weekly 8 -session worry intervention group $(n=7)$ or wait-list control $(n=6)$. Results: All but one participant completed measures at all time points. Participants attended an average of six therapy sessions. Conclusions: Recruitment, retention and therapy uptake were feasible. Observed treatment effects were in the expected direction, but may be diluted compared with one-to-one interventions.
\end{abstract}

Keywords: cognitive behavioural group therapy, worry intervention, persecutory delusions

\title{
Introduction
}

Persecutory delusions are erroneous beliefs that others intend to cause one harm. They are one of the most common psychotic experiences and can lead to social withdrawal, exacerbation of emotional distress, and substantially reduced quality of life (Freeman, 2016).

Traditional approaches to the treatment of psychosis adopt a diagnostic approach (e.g. focusing on 'schizophrenia') but these yield only modest effects. Recent advances demonstrating the independence of different psychotic experiences have resulted in a call to develop experience-specific models and treatments to improve outcomes. Freeman's (2016) cognitive model of persecutory delusions identifies six key evidence-based maintenance factors. Whilst studies have shown that targeting each factor individually is beneficial, the most robust evidence to date relates to worry. A high level of worry predicts the persistence of persecutory delusions and a large randomized control trial (RCT), the Worry Intervention Trial (WIT; Freeman et al., 2015), demonstrated that six sessions of high-intensity individual cognitive behavioural therapy (CBT) targeting worry led to significant improvements in both worry (effect size, Cohen's $d=0.5$ ) and persecutory delusions (effect size, Cohen's $d=0.5$ ). Two-thirds of the change in delusions was accounted for by changes in worry. Worry is therefore indicated as a contributory causal factor in persecutory delusions that is amenable to change.

Recent interest in group-based therapies for psychosis reflects the view that groups may offer particular benefits (e.g. learning from others in a normalizing environment; Newton et al., 2007) and that they could be a cost-effective alternative to individual therapy. Some evidence suggests they may be less clinically effective, however (Barrowclough et al., 2006). The current study was a small feasibility RCT conducted within routine clinical practice. The WIT intervention has not previously been delivered in a group format and our aim was to adapt the original manual for this purpose and test it to inform the potential next stages of trial development. Primary outcomes were recruitment rates, retention and therapy uptake. A secondary aim was to preliminarily inspect possible treatment effects, expecting that the worry intervention group would show evidence of a greater reduction in worry and persecutory delusions than the control group. 


\section{Method}

Detailed information about the trial can be found in the full version of this paper online.

\section{Study design and participants}

This wait-list controlled non-blind feasibility RCT tested an 8-week worry intervention group plus standard care versus standard care alone. Participants were adults recruited from community mental health teams in Oxleas NHS Foundation Trust. Inclusion criteria were: aged 18-65 years, current persecutory delusion (persisting $>6$ months), schizophrenia-spectrum diagnosis, and clinically significant worry ( $>45$ on the Penn State Worry Questionnaire (Behar et al., 2003)). Exclusion criteria included: primary diagnosis of alcohol/substance dependency, organic syndrome or learning disability, and acute symptomatic distress requiring hospital admission. Standardized measures of worry, paranoia and persecutory delusions were administered at baseline and at 8 and 12 weeks. Recruitment and assessments were conducted by trial therapists, L.I. and A.M. (clinical psychologists within the teams).

\section{Randomization}

Participants were randomized to the worry group (WG) or wait-list control (CG).

\section{Procedure}

The worry group had eight weekly 1.5 -hour sessions. 'Worry periods' and strategies for postponing worry were the main interventions. A key adaptation from the WIT trial was an increased number of sessions (eight versus six) to allow additional space for group discussion and processes (e.g. setting ground rules). Aspects of the original one-to-one intervention deemed unsuitable for group delivery were excluded. As in WIT, group facilitators offered between-session contact (e.g. telephone calls, texts and/or postcards) to provide support. After study completion, participants were invited to give feedback via focus groups led by a serviceuser researcher (S.P.).

\section{Outcomes measures and statistical analysis}

Primary outcomes were recruitment rate, participant retention and therapy uptake. Thresholds for feasibility were: recruitment rates exceeding $30 \%$ of those referred and $50 \%$ of those screened for eligibility, retention rates greater than $80 \%$ for each assessment time point, and therapy uptake of at least three-quarters of WG participants attending four or more therapy sessions. Secondary outcome measures of persecutory delusions, paranoia and worry were utilized and adjusted treatment difference and confidence intervals were estimated using a linear mixed-effects model. As this was a feasibility study, $p$-values are not reported.

\section{Results}

\section{Recruitment and retention}

Over an 18-week period, 41 individuals were referred, $25(61 \%)$ of whom proceeded to a screening assessment. Thirteen participants (32\% of those referred and 52\% of those assessed 
for eligibility) were recruited and randomized to WG $(n=7)$ or $\mathrm{CG}(n=6)$. Of those assessed for eligibility but not recruited, the majority did not meet the central inclusion criterion of having a persecutory delusion. The recruitment rate was considered good given the staff resource available for recruitment (the equivalent of one clinician, 0.5 days per week, across three teams). All but one participant completed assessments at all time points.

\section{Baseline and clinical characteristics}

The demographic details of the participants were similar across groups. The average age was 48 years, and most participants were single and living alone. All were unemployed. Most had a diagnosis of schizophrenia and were open to Recovery Services, but one participant in each pathway was recruited from the Assertive Outreach and Rehabilitation Team. Baseline assessments showed that participants had high levels of worry and persecutory ideation, and delusions that were distressing and preoccupying.

\section{Therapy uptake}

The mean number of therapy sessions attended was 5.7 (SD 1.3). All participants attended at least four sessions, with one attending all eight sessions, three attending six sessions, two attending five sessions, and one attending four sessions. Across the WG condition, 40 of 56 possible sessions were attended $(71 \%)$.

\section{Efficacy outcome}

Compared with CG, the treatment effect of CBT on worry was in the medium effect size range at 8 and 12 weeks (Table 1). The treatment effect on delusions was in the small effect size range at 8 weeks and no longer apparent at 12 weeks. The effect size found on general paranoia was 0 . However, in all outcomes wide confidence intervals spanning zero meant that the true direction of effect cannot be determined.

\section{Adverse events}

No adverse events were reported during the main trial period.

\section{Discussion}

It was feasible to recruit and retain participants. Commensurate with similar studies, onethird of those referred, and half of those assessed for eligibility, entered the trial. Initially the recruitment target was 20 participants but unforeseen job changes for the researchers limited available recruitment time. Over 4 months, 13 participants were recruited with a resource equivalent to one clinician for 0.5 days per week. The decision to proceed with this sample size was made due to concerns that participants may withdraw if there were longer delays between recruitment and intervention commencing. A larger study would benefit from external funding to increase resources and thus expedite the recruitment process.

Retention rates were high; all but one participant completed all assessments. Therapy uptake was also feasible. No WG participants dropped out and all completed at least four sessions 
Table 1. Scores for efficacy outcome measures

\begin{tabular}{lllll}
\hline $\begin{array}{l}\text { Outcome } \\
\text { measures }\end{array}$ & $\begin{array}{l}\text { Worry group } \\
(n=7)\end{array}$ & $\begin{array}{l}\text { Wait-list control } \\
(n=6)\end{array}$ & $\begin{array}{l}\text { Adjusted mean } \\
\text { difference }(95 \% \text { CI) }\end{array}$ & $\begin{array}{l}\text { Standardized } \\
\text { effect size }(d)\end{array}$ \\
\hline $\begin{array}{l}\text { Worry (PSWQ) } \\
\quad \text { Week 0 }\end{array}$ & $\begin{array}{l} \\
61.0(5.7)\end{array}$ & $62.5(12.90)$ & & \\
$\quad$ Week 8 & $58.9(10.8)$ & $66.0(12.73)$ & $-6.0(-15.4,3.4)$ & -0.5 \\
$\quad$ Week 12 & $58.4(9.3)$ & $67.1(8.26)$ & $-7.6(-15.7,0.6)$ & -0.6 \\
PSYRATS-delusions & & & & \\
$\quad$ Week 0 & $18.4(3.4)$ & $16.0(5.1)$ & $-2.2(-9.4,5.1)$ & -0.4 \\
$\quad$ Week 8 & $12.3(5.5)$ & $12.8(6.7)$ & $-0.6(-5.1,4.0)$ & -0.1 \\
$\quad$ Week 12 & $15.7(4.6)$ & $14.7(4.4)$ & & \\
Paranoia (GPTS-B) & & & & \\
$\quad$ Week 0 & $57.5(10.7)$ & $55.3(12.7)$ & $-0.2(-17.6,17.3)$ & -0.0 \\
$\quad$ Week 8 & $52.5(9.7)$ & $51.2(20.9)$ & $-0.8(-13.4,11.7)$ & -0.1 \\
$\quad$ Week 12 & $56.6(8.3)$ & $56.2(15.3)$ & & \\
\hline
\end{tabular}

Data are means $(S D)$. PSWQ, Penn State Worry Questionnaire; PSYRATS-delusions, Psychotic Symptoms Rating Scale_delusions; GPTS-B, Green Paranoid Thoughts Scale, Part B. Standardized effect sizes were calculated conservatively using pooled standard deviations from week 8 and week 12, respectively.

(average attendance was six of eight sessions). As three (of seven) participants (43\%) missed at least three sessions, however, some significant content was missed.

Focus group interviews with four participants revealed that the group was, overall, highly valued. There was a sense of the group targeting an important issue and being beneficial, with coping strategies developing, and self-esteem and activity levels improving. The importance of the group context was noted not only in terms of normalizing participants' experiences but also providing a supportive environment. Participants were unanimous that the number of group sessions felt too few, however.

Observed effect sizes were in the expected direction such that WG may have had a beneficial impact on worry at 8 and 12 weeks and on delusions at 8 weeks, but not 12 weeks. Confidence intervals were wide and spanned 0; a fully powered RCT would be required to adequately estimate the true size and direction of effect. The observed effect on worry may be partially linked to worry increasing in CG participants, a pattern not observed in the WIT trial. This could be due to environmental factors affecting this sample or simply chance given the small sample size. Overall, effect sizes were diluted from the individual delivery method, and changes in delusions did not translate into changes in general levels of paranoia. In the test of one-toone therapy in WIT (an assessor-blind trial), all primary and secondary outcome measures improved with the CBT intervention.

Participants and therapists agreed that future groups should be longer, and therapists felt that in-session time pressure had impacted on efficacy. They found it difficult to tailor techniques individually to each client, and balance the 'didactic' components (communicating key techniques) and the less structured supportive group discussions within the time. Increasing the intervention length (e.g. twelve 2-hour sessions) would help to address this, although getting the detailed individualized plans possible in individual therapy could still be difficult. Pacing sessions was also challenging, and so as to include everyone, the pace was slower than ideal for some participants. 
Future trials could consider incorporating a one-to-one therapy arm (rather than CG) and an economic evaluation. A full RCT of this design would allow comparative effectiveness and cost-effectiveness of group versus individual treatment to be established. This would be crucial before recommendations to adopt a group version of this intervention could be incorporated into clinical guidelines (especially given the gold-standard evidence that exists demonstrating the effectiveness of the individual approach; Freeman et al., 2015)

We suspect that, whilst at first glance groups seem like a less costly option, the need for a greater number of sessions, as well as the presence of two staff members may mean this is not the case. We felt the group format also decreases therapist flexibility in terms of scheduling sessions and hence may inadvertently increase the number of missed sessions.

Study limitations included a small sample size and the lack of an active control. Furthermore, the feasibility of recruiting to multiple groups in each arm was not established (which would be required in a larger trial) and the in-service nature of the study meant the therapists conducted the assessments and were unblind to treatment allocation. Feasibility to retain participants over a 1-month follow-up was established, but not whether retention could extend for a longer period (e.g. 6 months), which would be required in a full RCT.

This study provides an early indication that it may be feasible to run a larger trial to test the effectiveness of a group-based worry intervention for persecutory delusions. However, a larger scale pilot would need to test feasibility more extensively before a full RCT could be justified.

\section{Acknowledgements}

The authors would like to thank the participants and the clinical teams within Oxleas NHS Foundation Trust for their support of this study.

Ethical statement: The authors assert that all procedures contributing to this work comply with ethical standards of the relevant national and institutional committees on human experimentation and with the Helsinki Declaration of 1965, and its most recent revision.

Conflicts of interest: The authors have no conflicts of interest with respect to this publication.

Financial support: This work was supported by Oxleas NHS Foundation Trust.

\section{Supplementary material}

To view supplementary material for this article, please visit https://doi.org/10.1017/ S1352465818000383

\section{References}

Barrowclough, C., Haddock, G., Lobban, F., Jones, S., Siddle, R. O. N., Roberts, C. and Gregg, L. (2006). Group cognitive-behavioural therapy for schizophrenia. British Journal of Psychiatry, 189, $527-532$.

Behar, E., Alcaine, O., Zuellig, A. R. and Borkovec, T. D. (2003). Screening for generalized anxiety disorder using the Penn State Worry Questionnaire: a receiver operating characteristic analysis. Journal of Behavior Therapy and Experimental Psychiatry, 34, 25-43. https://doi.org/10.1016/ S0005-7916(03)00004-1 
Freeman, D. (2016). Persecutory delusions: a cognitive perspective on understanding and treatment. The Lancet Psychiatry, 3, 685-692. https://doi.org/10.1016/S2215-0366(16)00066-3

Freeman, D., Dunn, G., Startup, H., Pugh, K., Cordwell, J., Mander, H. et al. (2015). Effects of cognitive behaviour therapy for worry on persecutory delusions in patients with psychosis (WIT): a parallel, single-blind, randomised controlled trial with a mediation analysis. The Lancet Psychiatry, 2, 305-313. https://doi.org/10.1016/S2215-0366(15)00039-5

Newton, E., Larkin, M., Melhuish, R. and Wykes, T. (2007). More than just a place to talk: young people's experiences of group psychological therapy as an early intervention for auditory hallucinations. Psychology and Psychotherapy: Theory, Research and Practice, 80, 127-149. https: //doi.org/10.1348/147608306X110148 